\title{
Estigma de los profesionales de enfermería de salud mental hacia las personas con trastorno mental grave
}

Stigma of Mental Health Nursing Professionals Towards People with Severe Mental Disorder

\author{
Javier Sanz Calvo ${ }^{1}$ \\ 'Enfermero especialista en salud mental del Hospital Universitario Infanta Cristina. Parla, Madrid, España. \\ Contacto: jsanzc@salud.madrid.org \\ Fecha de recepción: 8 de junio de 2018 / Fecha de aceptación: 20 de septiembre de 2018
}

\begin{abstract}
Resumen
Introducción: Los profesionales de enfermería de salud mental se encuentran en contacto directo y continúo con las personas que padecen un trastorno mental grave. Por ello, son los encargados de prestar unos cuidados de calidad libres de prejuicios y ausentes de estigmatización. El objetivo del estudio es medir el grado de estigmatización de los profesionales de enfermería de salud mental del área sur de la Comunidad de Madrid hacia las personas diagnosticadas de trastorno mental grave.

Metodología: Se trata de un estudio descriptivo transversal dirigido a enfermeras que trabajen o hayan trabajado en algún recurso asistencial de salud mental en la zona sur de la Comunidad de Madrid (Leganés, Parla y Fuenlabrada, principalmente), teniendo una experiencia mínima de un mes. Para la recogida de los datos se utilizó el Cuestionario de Atribución-27.

Resultados: Se obtienen 65 participantes. Generalmente, las puntuaciones para las enfermeras son bajas. Se aprecia que, cuanto mayor es la experiencia profesional, entendida por tiempo trabajado $(p=0,02)$, variedad de recursos asistenciales $(p=0,01)$ y variación en la población, menor es el estigma sentido por las enfermeras hacia la enfermedad mental. Destaca la formación de las enfermeras especialistas en salud mental, respecto a las generalistas, por gozar de niveles significativamente menores en estigmatización $(p<0,01)$.

Conclusión: Las enfermeras de salud mental tienen puntuaciones bajas en estigma, aunque se siguen percibiendo marcados estereotipos hacia la patología mental. Tanto la experiencia profesional como, sobre todo, la formación especializada en salud mental, mejoran los

cuidados prestados hacia las personas con trastorno mental.
\end{abstract}

Palabras clave: estigma social, salud mental, enfermería, especialidades de enfermería, trastorno mental.

\begin{abstract}
Background: Mental health nursing professionals are in direct and continuous contact with people who have a severe mental disorder. Therefore, they are responsible for providing quality care without prejudice and stigmatization. The aim of this study is to measure the level of stigmatization of mental health nursing professionals in the south area of Madrid towards people diagnosed with severe mental disorder.

Method: This is a descriptive study about the nurses who work or have ever worked in any mental health care unit in the south of Madrid (Leganés, Parla and Fuenlabrada, mainly), with a mínimum of one month experience. The Attribution Questionnaire-27 was used to collect the information.

Results: 65 participants. Generally, the score for nurses are low. We can observe that the more professional experience, understood by time worked $(p=0,02)$, variety of care unit $(p=0,01)$ and variety in the population, the lower is the stigma felt by nurses towards the mental illness. It is highlights the formation of nurses specialized in mental health, compared to general nurses, for having significantly lower levels of stigmatization $(p<0,01)$.

Conclusion: Mental health nurses have low score in stigma, although the stereotypes towards mental pathology continue to exist. Both the professional experience and, specially, specialized formation in mental health, improve the care towards people with severe mental disorders.
\end{abstract}

Keywords: social stigma, mental health, nursing, specialties, nursing, mental disorder. 


\section{Introducción}

El concepto de enfermedad mental ha ido evolucionando a lo largo de la historia; sin embargo, la asociación de éste con peligrosidad, miedo o desconfianza ha sido constante durante años, hecho que ha favorecido al proceso de estigmatización de las personas que sufren un trastorno mental.

Actualmente, el estigma se podría definir como la posesión de algún atributo o característica que devalúa a la persona que lo tiene en un contexto social determinado. Es decir, los estigmas son características sociales sobre las cuales las demás personas poseen estereotipos, actitudes y creencias negativas, que acaban produciendo que estos individuos sean discriminados $\mathrm{y}$ excluidos $^{1,2}$.

Una definición muy aceptada es la que propone Goffman en 1963 en su publicación Estigma: la identidad deteriorada, donde define el fenómeno como "atributos negativos de un individuo que le generan un profundo descrédito y desvaloración social". Como consecuencia, estos atributos degradan y rebajan a las personas portadoras originando dificultades significativas en las relaciones sociales y en el propio bienestar de quienes lo padecen ${ }^{3,4}$.

Es importante destacar que existen diferentes tipos de estigma. Asimismo, el sociólogo Goffman describe tres tipos de estigma en 1963: el estigma físico referido a deformidades corporales visibles, el estigma de pertenencia o identidad grupal (etnia, nación, religión, etc.) y el estigma de carácter o personalidad que hace referencia a características personales que son interpretadas como defectos (identidad sexual, genero, trastorno mental, adicción...) $)^{3,5}$. En este artículo, nos centraremos únicamente en el estigma de carácter, ya que es el sufrido por los pacientes que padecen enfermedades mentales.

Los profesionales sanitarios, sobre todo el personal enfermero, son los encargados del cuidado directo a los enfermos mentales. Como hemos visto, estos pacientes poseen características diferentes al resto de usuarios de los servicios sanitarios, y es por ello por lo que las enfermeras debemos adaptarnos para ofrecerles una buena calidad asistencial. Por tanto, es imprescindible dejar atrás aquellas concepciones o actitudes negativas sobre los enfermos mentales que a menudo se observan en el ámbito asistencial, ya que nos impiden llegar a conseguir un cuidado de calidad.
Establecer una apropiada relación terapéutica entre el profesional enfermero y cualquier paciente es una herramienta básica de la que no podemos prescindir, aún más si se trata de un paciente con problemas de salud mental. Igualmente, está demostrado que los profesionales especializados en el área de salud mental, al poseer más formación y conocimientos sobre las patologías mentales, son capaces de conseguir un mayor acercamiento y una fructífera relación terapéutica con los afectados ${ }^{6}$.

Finalmente, hay que destacar que no solamente es de vital importancia que adquieran adecuadas relaciones de apoyo y conocimientos, sino que también ellas son las encargadas de realizar una correcta educación para la salud a los diferentes grupos sociales con la finalidad de evitar el proceso de estigmatización.

\section{Metodología}

\section{Diseño del estudio}

Se trata de un estudio descriptivo transversal.

\section{Población de estudio}

Enfermeras que trabajen o hayan trabajado en la red de salud mental (UHB, UCPP, UHTR y CSM, principalmente) de la zona sur de la Comunidad de Madrid (Leganés, Parla, Fuenlabrada, principalmente).

\section{Criterios de selección}

- Deben ser profesionales sanitarios que trabajen actualmente o hayan trabajado en el pasado en el ámbito de la salud mental.

- Haber realizado o realizar su profesión habitual en al menos uno de los recursos de asistencia mental de la zona sur de la Comunidad de Madrid.

- Tener al menos un mes de experiencia laboral como enfermera en la red asistencial psiquiátrica.

\section{Muestra}

Se decide realizar un muestreo no probabilístico por conveniencia. 


\section{Variables de estudio}

Variables independientes:

- Edad.

- Sexo.

- Tiempo trabajado en salud mental.

- Población de trabajo.

- Recurso de salud mental.

- Formación especializada en salud mental.

- Modalidad de adquisición de la formación especializada (solo en caso de tenerla): vía EIR (Enfermera Interno Residente) o vía extraordinaria.

\section{Variable dependiente:}

- Puntuación obtenida en el Cuestionario de Atribución-27 o Attribution Questionnaire-27 (AQ-27).

Para la recolección de datos se ha utilizado un cuestionario validado llamado Cuestionario de Atribución-27 (AQ-27), que podemos consultar en el Anexo I. Se trata de un cuestionario autoadministrado desarrollado por Corrigan, Markowitz, Watson, Rowan y Kubiak en 20037 y traducido al castellano gracias a Muñoz, Pérez, Crespo y Guillén en $2009^{8}$.

Este cuestionario fue diseñado para evaluar una serie de atribuciones o constructos (conductas, afectos, actitudes, etc.) de cualquiera hacia una hipotética persona diagnosticada de enfermedad mental grave (esquizofrenia). En resumen, fue creado para evaluar el estigma hacia las personas con patología mental y está compuesto por 27 preguntas con respuestas en una escala tipo Likert de 1 a 9.

Las 27 preguntas o ítems se agrupan en 9 factores (3 ítems para cada factor):

- Responsabilidad sobre la enfermedad mental: grado en el que las personas pueden controlar la causa de la enfermedad mental o ser responsables de su situación actual (ítems: $10+11+23$ ).

- Piedad o culpa: grado de preocupación (ítems: $9+22+27)$.

- Ira o enfado: cantidad de malestar o irritación (ítems: 1+4+12).

- Peligrosidad: percepción de que representan una amenaza para los demás (ítems: $2+13+18$ ).

- Miedo: nivel de terror expresado (ítems: 3+19+24).

- Ayuda: disposición a prestar ayuda (ítems: $8+20+21)$.

- Coacción: entendido como obligación a realizar un tratamiento adecuado (ítems: 5+14+25).
- Segregación: referido a la creencia de enclaustramiento de esta tipología de pacientes en hospitales psiquiátricos, de manera que estén separados de la sociedad (ítems: 6+15+17).

- Evitación: evitar vivir, trabajar o, simplemente, relacionarse cerca de una persona con enfermedad mental (ítems: 7+16+26).

De este modo, las puntuaciones de cada factor oscilan entre 3 y 27 . El resultado se expresaría: cuanto mayor puntuación del ítem, mayor será el valor de ese factor para la persona, excepto en el Factor 9 (Evitación) ya que las puntuaciones se invierten.

\section{Análisis de datos}

El tratamiento estadístico de los datos se realizó con el programa SPSS v.14® (SPSS Inc, Chicago, IL), mediante las pruebas no paramétricas de U MannWhitney y Kruskal-Wallis.

\section{Resultados}

Se consiguieron un total de 58 profesionales de enfermería (Tabla 1), la mayoría de los encuestados eran mujeres $(79 \%)$ y la edad media se encuentra en 38,32 años.

Además, la media del tiempo trabajado en los diferentes dispositivos de la red de salud mental es de 8,6 años, es decir 8 años y 8 meses. A partir de este dato extraemos que dos tercios de la población encuestada $(65,5 \%)$ posee una experiencia menor a 10 años y que el $40 \%$ tiene una experiencia en estos servicios de tan solo 3 años o menos.

En cuanto a la población de trabajo, la mayoría pertenecen a Leganés $(65,5 \%$ ), seguido por Parla.

El recurso asistencial más participativo es la Unidad de Hospitalización Breve (UHB) de psiquiatría. A continuación, y en la misma situación, se encontrarían la Unidad de Cuidados Psiquiátricos Prolongados (UCPP) y la Unidad Hospitalaria de Tratamiento y Rehabilitación(UHTR).

Una parte esencial es evaluar el grado de formación de los profesionales de enfermería, es decir la especialización en materia de salud mental. Según los datos, los profesionales especialistas alcanzan alrededor del $40 \%$ frente a la mayoría de la muestra que no posee el título de Enfermera Especialista en Salud Mental. 


\begin{tabular}{|lc|}
\hline \multicolumn{2}{|l|}{\begin{tabular}{l} 
N $=\mathbf{5 8}$ \\
\hline Mujeres
\end{tabular}} \\
\hline Varones & 79 \\
\hline Edad (\%) & 21 \\
\hline $18-39$ años & 51,7 \\
\hline 40-64 años & 48,3 \\
\hline Edad Media (años) & 38,32 \\
\hline Tiempo Trabajado (\%) & 39,6 \\
\hline S3 años & 25,9 \\
\hline$>3$ años y<10 años & 34,5 \\
\hline$\geq 10$ años & 8,6 \\
\hline Tiempo Trabajado Medio (años) & 65,5 \\
\hline Población de Trabajo (\%) & 31 \\
\hline Leganés & 8,6 \\
\hline Parla & \\
\hline Fuenlabrada & 60,3 \\
\hline Recurso de Trabajo (\%) & 43,1 \\
\hline Unidad de Hospitalización Breve (UHB) & 39,7 \\
\hline Unidad de Cuidados Psiquiátricos Prolongados (UCPP) & 25,8 \\
\hline Unidad Hospitalaria de Tratamiento y Rehabilitación (UHTR) & 24,1 \\
\hline Hospital de Día -Adultos e Infanto-juvenil- (HD) & 12,1 \\
\hline Centro de Salud Mental (CSM) & \\
\hline Centro de Atención Integral a Drogodependencias (CAID) \\
\hline Fuente: elaboración propia. & \\
\hline
\end{tabular}

Tabla 1. Características sociodemográficas de los profesionales de enfermería de salud mental de la zona sur de la Comunidad de Madrid.

\section{GRADO DE FORMACIÓN}

Especialistas = No Especialistas

Gráfico 1.

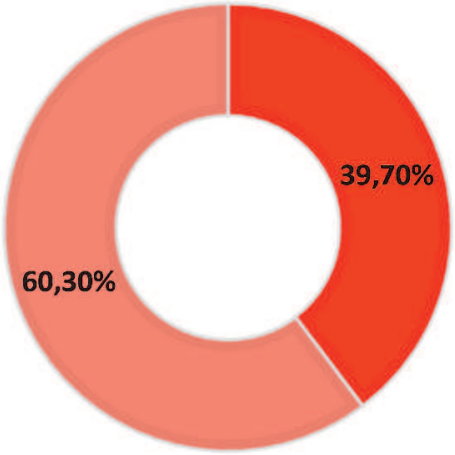

Fuente: elaboración propia.

\section{VÍA ESPECIALIZACIÓN}

E.I.R Extraordinaria

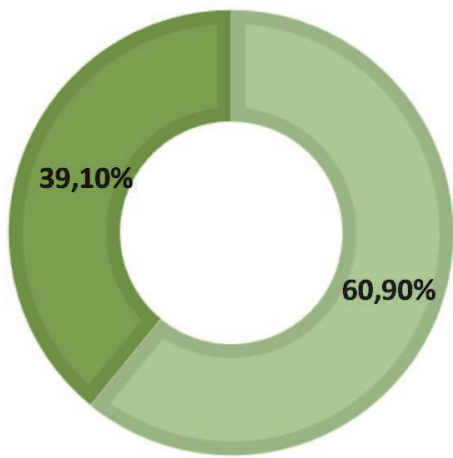

Gráfico 2.

Fuente: elaboración propia. 


\begin{tabular}{|c|c|}
\hline İtems (rango 1-9) / Factor (rango 3-27) & MEDIA $\left(D^{* *}\right)$ \\
\hline FACTOR 1: RESPONSABILIDAD SOBRE LA ENFERMEDAD MENTAL & $11,03(3,80)$ \\
\hline 10. Pensar que es su culpa & $1,48(1,47)$ \\
\hline 11. Ser controlable la causa de la situación & $6,00(1,96)$ \\
\hline 23. Responsabilidad sobre la situación actual & $3,55(2,07)$ \\
\hline FACTOR 2: PIEDAD/CULPA & $18,4(3,40)$ \\
\hline 9. Sentir pena & $4,52(2,00)$ \\
\hline 22. Compresión & $7,41(1,23)$ \\
\hline 27. Grado de preocupación & $6,47(1,78)$ \\
\hline FACTOR 3: IRA/ENFADO & $5,75(3,01)$ \\
\hline 1. Sentirse violentado & $2,03(1,44)$ \\
\hline 4. Sentirse enfadado & $1,43(1,01)$ \\
\hline 12. Sentirse irritado & $2,29(1,53)$ \\
\hline FACTOR 4: PELIGROSIDAD & $7,56(4,67)$ \\
\hline 2. Sentirse inseguro & $2,59(1,80)$ \\
\hline 13. Considerarlo peligroso & $2,86(1,91)$ \\
\hline 18. Sentirse amenazado & $2,12(1,70)$ \\
\hline FACTOR 5: MIEDO & $5,25(3,49)$ \\
\hline 3. Aterrorizado & $1,43(0,94)$ \\
\hline 19. Asustarse & $1,98(1,46)$ \\
\hline 24. Grado en que le aterroriza & $1,84(1,41)$ \\
\hline FACTOR 6: AYUDA & $24,05(2,73)$ \\
\hline 8. Dispuesto a hablar con él sobre su problema & $8,28(1,28)$ \\
\hline 20. Probabilidad de ayudarle & $8,14(0,96)$ \\
\hline 21. Certeza de ayudarle & $7.64(1,27)$ \\
\hline FACTOR 7: COACCIÓN & $17,51(5,48)$ \\
\hline 5. Exigirle que tome la medicación & $7,34(1,92)$ \\
\hline 14. Debe ser obligado a ponerse en tratamiento médico & $6,43(2,42)$ \\
\hline 25. Si estuviera a su cargo, obligarle a vivir en piso supervisado & $3,74(2,45)$ \\
\hline FACTOR 8: SEGREGACIÓN & $8,34(5,18)$ \\
\hline 6. Supone un riesgo si no se le hospitaliza & $3,64(2,28)$ \\
\hline 15. Mejor recluirlo en un hospital psiquiátrico & $2,22(1,96)$ \\
\hline 17. El hospital psiquiátrico es el mejor lugar para él & $2,48(1,85)$ \\
\hline FACTOR 9: EVITACIÓN * & $16,65(5,79)$ \\
\hline 7. Si fuera empresario, lo entrevistaría para un trabajo * & $6,05(2,29)$ \\
\hline 16. Compartiria coche * & $5,33(2,67)$ \\
\hline 26. Si fuera propietario, le alquilaría un piso * & $5,28(2,34)$ \\
\hline \multicolumn{2}{|c|}{$\begin{array}{l}\text { * Las puntuaciones para estos ítems y factor están invertidas respecto el resto. Es decir, a } \\
\text { mayor puntuación, menor evitación. } \\
{ }^{* *} \text { Desviación típica. }\end{array}$} \\
\hline
\end{tabular}

Tabla 2: Medias y desviaciones típicas de las puntuaciones para cada ítem y factor del Cuestionario de Atribución 27 en profesionales de enfermería. 
Dentro de este dato de profesionales especialistas que han participado, podemos diferenciar dos categorías según la vía para obtener el título: vía extraordinaria o vía EIR (Enfermero Interno Residente). Encontramos que la vía de especialización EIR parece algo más participativa $(\mathrm{n}=14)$ que la extraordinaria $(\mathrm{n}=9)$.

En general, las puntuaciones del AQ-27 son bajas (Tabla 2), lo cual es un buen indicador del nivel de estigma percibido por los profesionales de enfermería hacia los pacientes con enfermedades mentales. Tanto es así, que las puntuaciones obtenidas respecto a los sentimientos de ira o enfado hacia el paciente, la peligrosidad percibida de este y el miedo son ínfimas (Factor 3:5/27, Factor 4: 7/27 y Factor 5: 5/27).

Además, nuestra muestra posee altos niveles de disponibilidad de ayuda (Factor 6: 24/27), datos que no resultan alarmantes ya que este colectivo se dedica al cuidado directo de personas. Entre otros datos favorecedores encontramos que poseen medio-altos niveles de preocupación por las personas aquejadas de trastornos mentales (Factor 2: 18/27). También, al tratarse de profesionales encargados del tratamiento farmacológico del paciente, encontramos niveles medio-altos en la coacción u obligación a recibir un tratamiento adecuado (Factor 7: 17/27).

Por lo tanto, en líneas generales, las puntuaciones medias de este cuestionario para los profesionales de enfermería son muy buenas y esperanzadoras.

Observando los datos presentes en el Gráfico 3, podemos afirmar que apenas existen diferencias entre el género y la edad de los profesionales sanitarios.

Tan solo podemos observar que el grupo de profesionales más joven ( $\leq 30$ años) obligan en menor frecuencia a llevar un tratamiento adecuado o coaccionar a los enfermos mentales respecto al grupo de profesionales más veteranos ( $>30$ años), teniendo estos últimos cifras mayores en coacción $(\mathrm{p}=0,02)$.

Gráfico 3. GÉNERO Y EDAD

\begin{tabular}{|c|c|c|c|c|c|c|c|c|c|}
\hline & $\square$ Media & 로 Masculinc & \multicolumn{2}{|c|}{$\equiv$ Femenino } & -30 años & $\equiv 31-40$ año & \multicolumn{2}{|c|}{$\equiv 41-61$ años } & \\
\hline \multicolumn{10}{|l|}{25,00} \\
\hline \multicolumn{10}{|l|}{20,00} \\
\hline \multirow{2}{*}{\multicolumn{10}{|c|}{15,00}} \\
\hline & & & & & & & & & \\
\hline \multicolumn{10}{|l|}{5,00} \\
\hline \multirow{2}{*}{0,00} & & & & & & & & & \\
\hline & $\begin{array}{c}\text { Responsab } \\
\text { ilidad }\end{array}$ & Piedad & Ira & Peligro & Miedo & Ayuda & Coacción & $\begin{array}{c}\text { Segregació } \\
n\end{array}$ & Evitación \\
\hline$\square$ Media & 11,03 & 18,40 & 5,76 & 7,57 & 5,26 & 24,05 & 17,52 & 8,34 & 16,66 \\
\hline E Masculino & 12,33 & 17,08 & 6,92 & 6,17 & 5,17 & 23,67 & 16,67 & 7,00 & 18,67 \\
\hline 䓃 Femenino & 10,70 & 18,74 & 5,46 & 7,93 & 5,28 & 24,15 & 17,74 & 8,70 & 16,13 \\
\hline$\equiv 22-30$ años & 9,50 & 18,44 & 5,50 & 6,72 & 5,50 & 24,89 & 15,11 & 6,89 & 17,39 \\
\hline 量 $31-40$ años & 12,00 & 18,32 & 6,16 & 8,42 & 5,37 & 23,68 & 20,05 & 9,16 & 17,32 \\
\hline E $41-61$ años & 11,48 & 18,43 & 5,62 & 7,52 & 4,95 & 23,67 & 17,29 & 8,86 & 15,43 \\
\hline
\end{tabular}

Fuente: elaboración propia. 
Respecto al tiempo trabajado en la red de salud mental, afirmamos que los participantes más novatos (experiencia inferior o igual a 2 años) perciben más miedo hacia el paciente con trastorno mental que cualquier otro grupo $(p=0,02)$. Sin embargo, la muestra más experimentada (mayor o igual a 10 años) tiene niveles muy bajos en el factor miedo. Por lo que se puede deducir que los profesionales con más experiencia tienen menos percepción de miedo hacia las perso- nas con enfermedad mental.

En cuanto a la población donde se realizó o se realiza el trabajo de los profesionales de enfermería, nos encontramos que las puntuaciones no son muy dispares entre sí. De esta manera, los factores más destacables en los que se encuentran datos más alejados de la media son Peligrosidad, Coacción y Segregación (Tabla 3).

\begin{tabular}{|l|c|c|c|}
\hline \multicolumn{4}{|c|}{ Tabla 3. Medias de los factores según poblaciones de trabajo. } \\
\hline \multicolumn{1}{|c|}{ Factor (rango 3-27) } & 4. Peligrosidad & $\mathbf{7 . ~ C o a c c i o ́ n ~}$ & $\mathbf{8 . ~ S e g r e g a c i o ́ n ~}$ \\
\hline MEDIA & $\mathbf{7 , 5 7}$ & $\mathbf{1 7 , 5 2}$ & $\mathbf{8 , 3 4}$ \\
\hline Leganés (65\%) & 7,97 & 17,09 & 8,88 \\
\hline Parla (31\%) & 8,07 & $\mathbf{2 0}$ & 8,93 \\
\hline Fuenlabrada (8\%) & $\mathbf{4 , 3 3}$ & $\mathbf{1 4 , 6 7}$ & $\mathbf{4 , 6 7}$ \\
\hline 1 población & 7,67 & 17,71 & 8,52 \\
\hline Varias poblaciones & 6,67 & $\mathbf{1 5 , 8 3}$ & $\mathbf{6 , 8 3}$ \\
\hline Fuente: elaboración propia. & \multicolumn{4}{|}{} \\
\hline
\end{tabular}

Tanto Leganés como Parla tienen unos valores muy similares entre sí y a la media de la muestra, lo que podemos traducir en unos niveles globales bajos de estigma percibidos hacia el paciente con trastorno mental. En esta ocasión, destaca Parla como el lugar de trabajo donde se coacciona con mayor frecuencia para llevar un tratamiento adecuado respecto al resto de poblaciones $(\mathrm{p}=0,05)$.

Además, comparando aquellos profesionales que solamente trabajaron en una ciudad frente a los que han tenido mayor diversidad en su ámbito laboral, se encuentra que los profesionales de enfermería con varias poblaciones distintas de trabajo demuestran tener mejores puntuaciones en estigma mental. Por lo tanto, podemos deducir a priori que la variedad en los lugares de empleo mejora la visión de los profesionales sobre sus pacientes, aunque estas diferencias no son relevantes.

Respecto a los recursos asistenciales de la red de salud mental (Gráfico 4), quizá uno de los más destacados es la Unidad de Cuidados Psiquiátricos Prolongados (UCPP) ya que tiene valores de estigmatización mucho más altos que el resto de los recursos. De esta forma, la UCPP tiende a percibir mayor miedo y a expresar más cantidad de ira hacia los pacientes con enfermedad mental. Esta afirmación puede venir respaldada por el hecho de que los profesionales que trabajan en la UCPP respecto al resto piensan que los enfermos mentales son más peligrosos. También demuestran tener puntuaciones más elevadas en segregación, es decir, en la idea de enclaustramiento en residencias de esta tipología de pacientes. Todo ello hace que estos profesionales tiendan a evitar con mayor frecuencia relacionarse con personas diagnosticadas de patología mental. No obstante, se trata de puntuaciones neutras y no significativas. Tan solo podemos afirmar que la UCPP obliga más que el resto de los dispositivos a llevar un tratamiento adecuado, ya que encontramos diferencias significativas en el Factor Coacción $(\mathrm{p}=0,02)$.

Por otra parte, recursos como las Unidades de Hospitalización Breve (UHB) o las Unidades Hospitalarias de Tratamiento y Rehabilitación (UHTR) obtienen puntuaciones más esperanzadoras. 
Gráfico 4. RECURSO DE TRABAJO

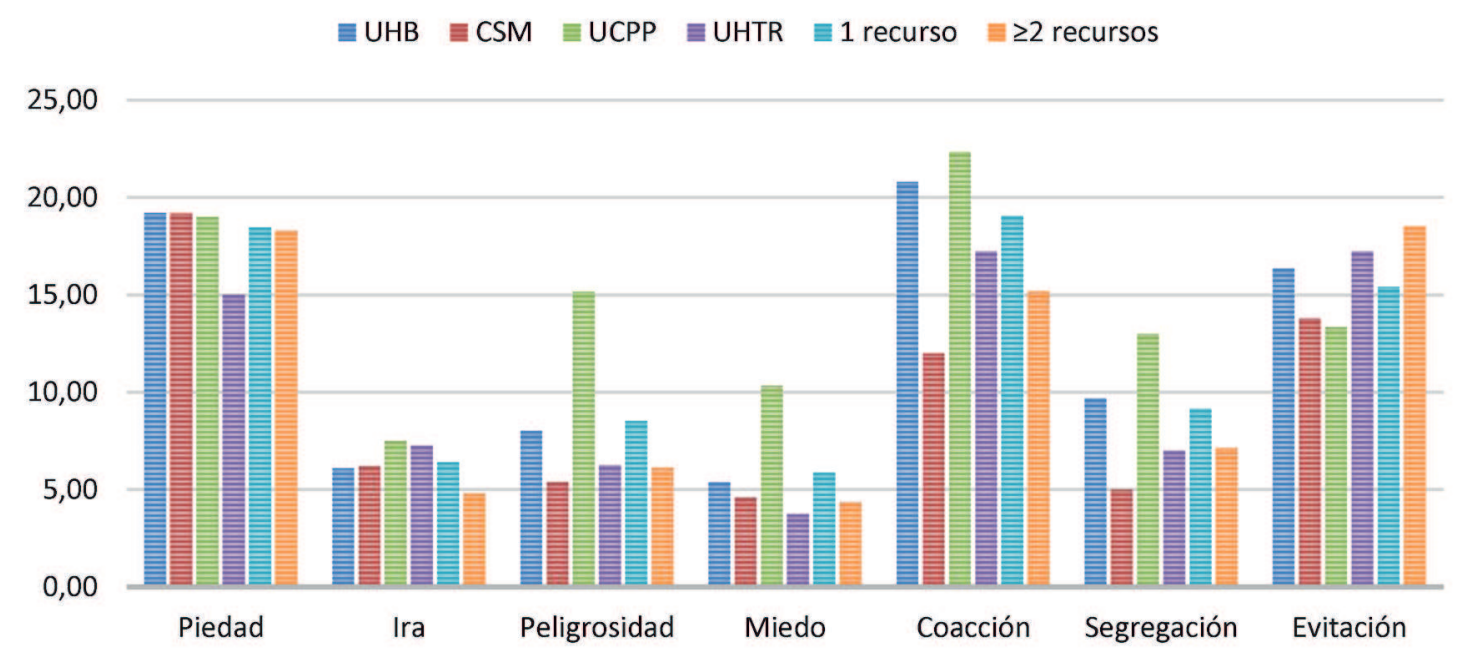

Fuente: elaboración propia.

En la misma línea se encuentran los Centros de Salud Mental (CSM) por ser unos de los mejores puntuados, siendo además los recursos con menos sensación de peligrosidad del paciente, menor segregación de éste hacia un internamiento y menor coacción del tratamiento. Sin embargo, no todo son datos favorables ya que los profesionales de los CSM tienden a evitar en más ocasiones relacionarse en su vida cotidiana con las personas que padecen trastornos mentales. Sin embargo, tampoco encontramos niveles de significación destacables.

Por último, se puede apreciar en el Gráfico 4 que existe una distinción entre profesionales que solamente ejercen o ejercieron su trabajo en un único recurso y aquellos que lo hicieron en varios ( $\geq 2$ recursos). De esta forma, se observa que los valores para los profesionales que pasaron por varios recursos son más bajos y por tanto expresan menor estigma que aquellos que tan solo se desarrollaron en un recurso, pero estos datos no son relevantes significativamente. No obstante, podemos afirmar que los profesionales que trabajaron en varios dispositivos obligan en menor cantidad al paciente, ya que encontramos niveles de significación en el factor Coacción $(p=0,01)$. Por ello, parece aconsejable que exista una variedad en la experiencia profesional dentro de los recursos asistenciales de salud mental.
Un objetivo del estudio es ver la importancia de la formación. Hasta ahora, hemos visto que los profesionales con más experiencia laboral tienen mejores puntuaciones, y queremos comprobar si aquellos con más experiencia académica también tienen mejores puntuaciones.

Asimismo, según los datos reflejados en el Gráfico 5 , los profesionales en contacto con los pacientes de salud mental sin título de especialista (generalistas) poseen puntuaciones mayores en Peligrosidad, Coacción y Segregación. Es decir, obligan más a llevar un tratamiento adecuado y a favorecer el internamiento psiquiátrico de estos pacientes porque los perciben como más peligrosos, al contrario que las profesionales enfermeras especialistas, con un alto nivel de significación $(\mathrm{p}<0,01)$.

Con estas afirmaciones queda comprobado que tanto la experiencia profesional (nivel de tiempo trabajado, variedad de poblaciones y recursos visitados) como la académica, son dos herramientas importantes, básicas y muy útiles para el correcto manejo de las personas que sufren una enfermedad mental. Destacando en esta última la especialización en salud mental de las enfermeras como algo recomendable y provechoso, tanto para los pacientes como para los profesionales. 
Gráfico 5.

\section{FORMACIÓN}

पGeneralistas I|| Especialista v. EIR IIII Especialista v. Extraordinaria

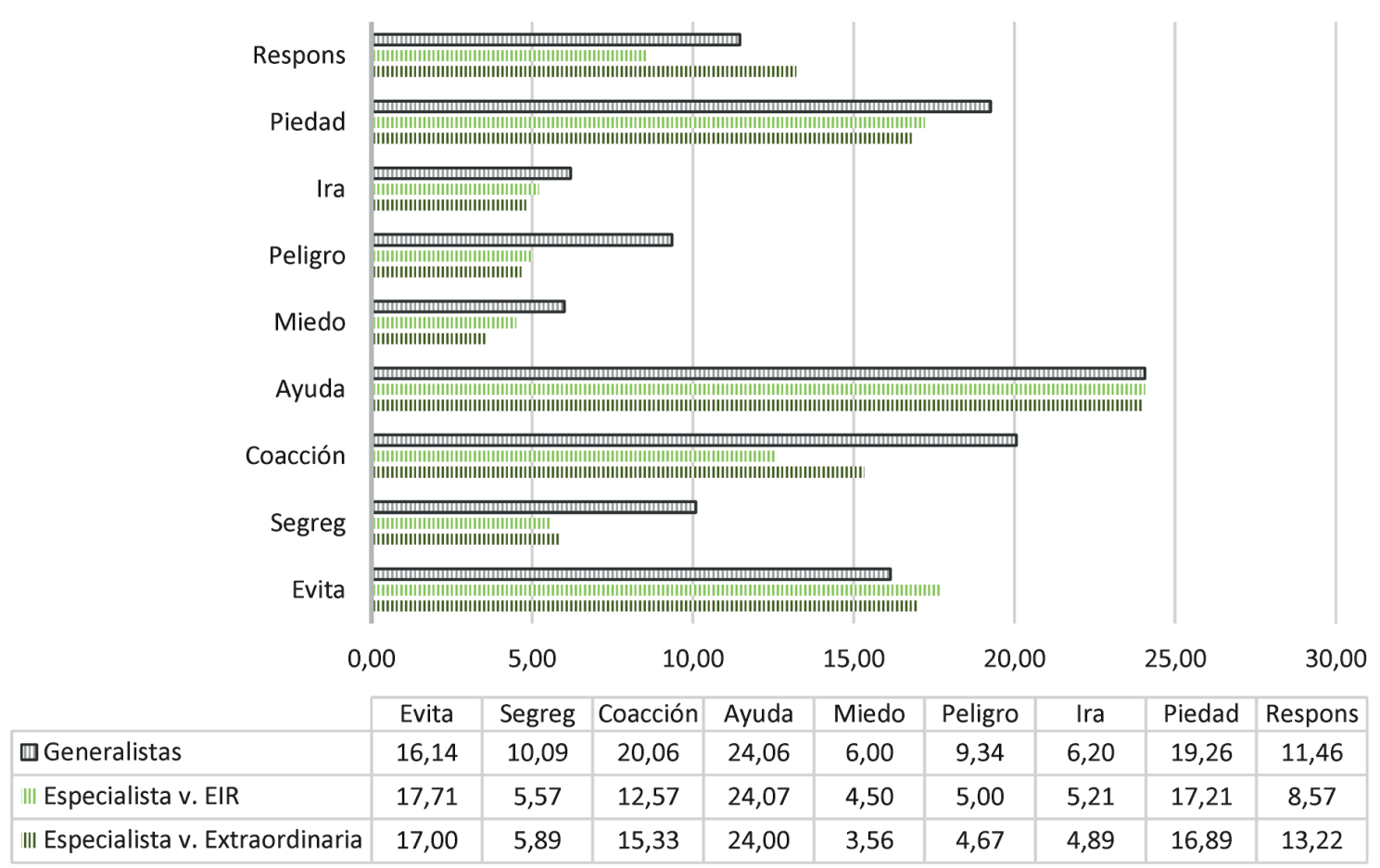

Fuente: elaboración propia.

Por otra parte, la única distinción significativa entre las diferentes vías de obtener la especialización reside en que la formación EIR consideran que los enfermos mentales son menos responsables de su patología que la muestra con formación especializada por vía extraordinaria $(\mathrm{p}<0,01)$.

\section{Discusión}

Según la OMS, la esquizofrenia es un trastorno mental que afecta alrededor de 21 millones de personas de todo el mundo (6000 millones) ${ }^{9}$. Según López-Ibor (2000) y Fischer et al. (2017), la prevalencia de esquizofrenia se acerca al $1 \%$ a nivel internacional ${ }^{1}$.

Asimismo, programas como " 1 decada4" desarrollados por la Junta de Andalucía, nos recuerdan que 1 de cada 4 personas padece una enfermedad mental a lo largo de su vida ${ }^{12}$.
Otros proyectos como "Obertament", originado en Cataluña, nos ofrecen datos similares: "1 de cada 5 jóvenes vive un problema de salud mental"13. En la misma línea se encuentra la OMS, ya que habla sobre que al menos el $20 \%$ de los jóvenes padecerá alguna forma de enfermedad mental ${ }^{14}$.

Todos estos argumentos se exponen para concienciar a la población de que las enfermedades de salud mental son un problema común y de gran magnitud que todos podemos experimentar.

Por otra parte, encontramos otros artículos similares al nuestro que exploran las actitudes de los profesionales sanitarios de salud mental.

Así Tay et al. (2004) manifiesta que la cualificación profesional avanzada y una experiencia de más de 10 años en el ámbito psiquiátrico favorecen actitudes significativamente más positivas ${ }^{15}$. 
Por su parte, el Instituto Andaluz de Salud Mental (1988) destaca que las personas que mostraban una actitud global más favorable eran los profesionales sanitarios con una edad inferior a 45 años y con una mayor categoría profesional ${ }^{16}$.

Como se refleja de los anteriores estudios, la cualificación avanzada o mayor categoría profesional, llamada en este proyecto formación especializada, influye directamente en las actitudes de los profesionales. En la misma línea se encuentra nuestro estudio ya que revela que existen grandes diferencias significativas entre enfermeras generalistas y especialistas, siendo estas últimas mejor valoradas.

Por tanto, diversos autores apuestan por esta premisa de "a mayor formación y experiencia laboral, menor estigma encontrado".

De esta manera, revisando el trabajo realizado por el Royal College of Psychiatrists de Londres (Mukherjee et al., 2002) encontramos que los médicos, no específicamente profesionales en la salud mental, tendían a creer que las personas con enfermedad mental eran peligrosas e impredecibles, pero este estigma parecía disminuir a medida que aumentaba la formación y experiencia profesional ${ }^{17}$.

Otro ejemplo, serían los numerosos estudios coincidentes en que las enfermeras de salud mental comparten estereotipos y manifiestan actitudes menos favorables en comparación con los psiquiatras, los cuales presentan una mejor formación, mayor conocimiento y opiniones más positivas ${ }^{15,16,18}$.

Finalmente, para visualizar mejor los datos del presente artículo, lo comparamos con otros estudios nacionales (Muñoz et al., 2009) realizados con una metodología similar en población general (Gráfico 6).

De esta forma, se observa en el Gráfico 6 que nuestra población enfermera tiene actitudes menos estigmatizantes que la población general en todos los factores. Este dato favorece gratamente la calidad de los cuidados ofrecidos por enfermería hacia las personas con enfermedad mental.

\section{Gráfico 6. Análisis comparativo de las puntuaciones en el Cuestionario de Atribucción-27 entre los profesionales de enfermería de salud mental y la población general de la Comunidad de Madrid.}

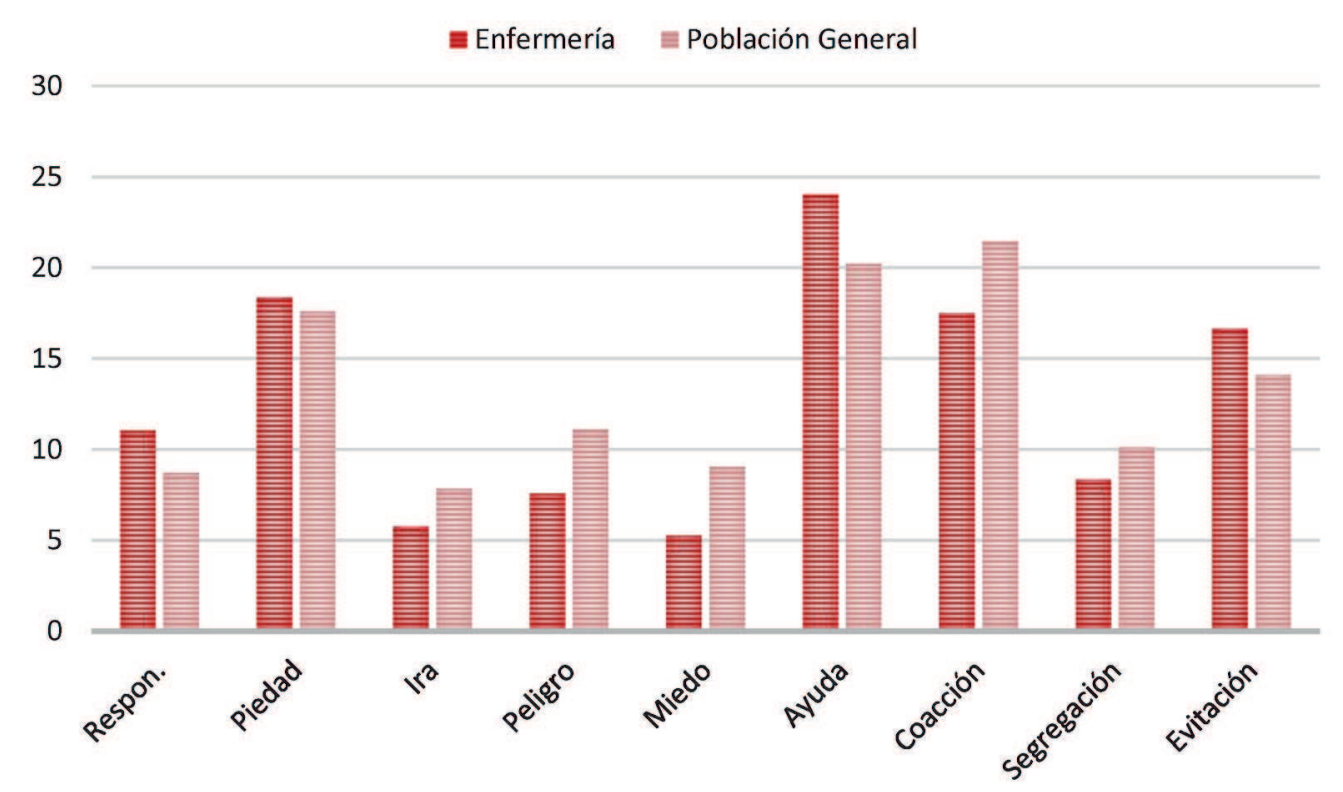

Fuente: Muñoz et al. $2009^{8}$ y elaboración propia. 
Además, datos aportados por otros trabajos sugieren que, globalmente, las actitudes de los profesionales que atienden a personas con enfermedad mental son sustancialmente más positivas que las de la población general, aunque siguen apareciendo ciertas actitudes estigmatizadoras ${ }^{18}$.

Finalmente, aportar que quizá los menores estereotipos presentados por los profesionales sanitarios respecto a otras poblaciones se deban al mejor conocimiento y acercamiento a las personas con esta tipología de enfermedad. Igualmente, Frías et al. (2017) o Sayols-Villanueva et al. (2015) sugieren que el contacto directo con personas con trastorno mental, así como el mayor grado de conocimiento de su patología, reducen significativamente los niveles de estigma en cualquier población ${ }^{21}$.

\section{Conclusión}

Históricamente el estigma se ha asociado a todas aquellas personas con cualidades que las hacían distintas, hecho que se acrecentaba en las personas que sufrían una patología mental. Actualmente, podemos señalar que siguen existiendo importantes estereotipos y prejuicios acerca de la enfermedad mental y de las personas que la padecen.

De esta manera, este estigma mental ocasiona numerosas consecuencias en toda clase de ámbitos, incluido el sanitario, lo que conduce a la discriminación. Asimismo, los pacientes con enfermedad mental se encuentran muy vinculados a los diferentes recursos de la red y, por tanto, a los profesionales sanitarios que la atienden, pero continúan existiendo hoy en día estereotipos marcados hacia la patología psiquiátrica desde las instituciones sanitarias. Sin embargo, se ha conseguido evolucionar en esta percepción.

Según los resultados del estudio, las enfermeras como gestoras del cuidado del enfermo mental demuestran tener actitudes menos estigmatizantes que la población general.

También queda comprobado que la experiencia laboral y académica de los profesionales de enfermería mejora la percepción hacia el paciente, reduciendo el estigma mental.

Igualmente, se hace hincapié en la importancia de la formación especializada en salud mental con el objetivo de mejorar los cuidados prestados, evitando la estig- matización.

\section{Bibliografía}

1. Sanjuan AM. El estigma de los trastornos mentales: discriminación y exclusión social. 2011.

2. Dovidio JF, Major B, Crocker J. Stigma: Introduction and overview. 2000.

3. Goffman E. Stigma: Notes on the management of spoiled identity. New York: Simon and Schuster; 2009.

4. Mascayano Tapia F, Lips Castro W, Mena Poblete C, Manchego Soza C. Estigma hacia los trastornos mentales: características e intervenciones. Salud mental. 2015;38: 53-58.

5. Cazzaniga Pesenti J, Suso Araico A. Salud mental e inclusión social: situación actual y recomendaciones contra el estigma. Confederación SALUD MENTAL ESPAÑA. 2015.

6. Castro-Morales JM, Cano-Molina V. Actitud del personal de enfermería ante el paciente psiquiátrico en unidades de segundo nivel de atención. Salud en Tabasco. 1997;3(1).

7. Corrigan P, Markowitz FE, Watson A, Rowan D, Kubiak MA. An attribution model of public discrimination towards persons with mental illness. J Health Soc Behav. 2003; 44(2): 162-179.

8. Muñoz M, Pérez E, Crespo M, Guillén Al. Estigma y Enfermedad Mental. Análisis del rechazo social que sufren las personas con enfermedad mental. Madrid: Editorial Complutense; 2009.

9. Organización Mundial de la Salud. Trastornos mentales [Internet]. Ginebra: OMS; 2017. [citado 18 ago 2017]. Disponible en: http://www.who.int/mediacentre/factsheets/fs396/es/.

10. Fischer BA, Buchanan RW. Schizophrenia in adults: epidemiology and pathogeneses. In: UpToDate, Rose, BD (Ed), UpToDate, Waltham, MA, 2017.

11. López-Ibor JJ, Cuenca O. La esquizofrenia abre las puertas. Programa de la Asociación Mundial de Psiquiatría para combatir el estigma y la discriminación debidos a la esquizofrenia. Vol 2. Madrid: Aula Médica; 2000.

12. 1 decada4.es [Internet]. Sevilla: Junta de Andalucia; 2004 [citado 4 ago 2017]. Disponible en: http://www.1decada4.es/.

13. Obertament.org [Internet]. Barcelona: Obertament; 2010 [citado 12 ago 2017]. Disponible en: https://obertament.org/es/.

14. Organización Mundial de la Salud. Salud de los adolescentes [Internet]. Ginebra: OMS; 2017. [citado 12 ago 2017]. Disponible en:

http://www.who.int/features/factfiles/adolescent_health/facts/ es/index4.html.

15. Tay SEC, Pariyasami SD, Ravindran K, Ali MIA, Rowsudeen MT. Nurses' attitudes toward people with mental illnesses in a psychiatric hospital in Singapore. Journal of psychosocial nursing and mental health services. 2004; 42(10): 40-47.

16. Instituto Andaluz de Salud Mental de Andalucía. Actitudes de la población andaluza ante los enfermos mentales. Sevilla: Instituto Andaluz de Salud Mental; 1988.

17. Mukherjee R, Fialho A, Wijetunge A, Checinski K, Surgenor T. The stigmatisation of psychiatric illness. The Psychiatrist. 2002; 26(5): 178-181.

18. Magliano L, De Rosa C, Fiorillo A, Malangone C, Guarneri M, Marasco $\mathrm{C}$, et al. Beliefs of psychiatric nurses about schizophrenia: a comparison with patients' relatives and psychiatrists. International Journal of Social Psychiatry. 2004; 50(4):319-330.

19. Frías VM, et al. Estigma: la relevancia del contacto social en el trastorno mental. Enferm Clin. 2017.

20. Sayols-Villanueva M, Ballester-Ferrando D, Gelabert-Vilella S, Fuentes-Pumarola C, Rascón-Hernán C. El estigma de los trastornos mentales en los estudiantes de enfermería. Rev de enfermería y salud mental. 2015; (2): 7-15.

21. Cebollero-Rodríguez M, Vaquero-Crespo C, López-Escudero MÁ, Saiz-Galdós J. Intervención contra el estigma hacia personas con enfermedad mental en jóvenes de institutos del Sur de la Comunidad de Madrid. 
Anexo I: Cuestionario de Atribución 27

\section{AQ-27 (Cuestionario de atribución 27).}

¡Hola!, bienvenido.

Muchas gracias por participar en esta encuesta. Solo te llevará un par de minutos.

Si usted rellena este cuestionario, está autorizando a que sus datos sean utilizados de forma ANÓNIMA con fines investigadores.

A continuación, unas sencillas preguntas:

* Obligatorio.

\section{1. ¿Cuál es tu número de colegiado? *}

Esta información es para asegurar la ausencia

de duplicidad de respuestas, y no será utilizada

con otros fines ya que el cuestionario es

ANÓNIMO.

\section{2. Edad. *}

\section{3. Sexo. *}

Marca solo un óvalo

\section{Masculino.}

Femenino.

\section{4. Tiempo trabajado en salud mental. *}

Expresado en meses y/o años ( 6 meses

o 2 años y 3 meses) de forma aproximada.

*Damos por hecho que usted es enfermera/o y que

trabaja o ha trabajado en contacto con salud mental.

Si no es así, no debería rellenar este formulario.

\section{5. Población de trabajo. *}

Lugar o lugares dónde se realizan o se han realizado la profesión relacionada con salud mental. *Damos por hecho que usted es enfermera/o y que trabaja o ha trabajado en contacto con salud mental. Si no es así, no debería rellenar este formulario.

Selecciona todos los que correspondan.

Leganés.

Parla.

Fuenlabrada.

Otro: 


\section{6. Recurso de trabajo. *}

Unidades o recursos dónde se realizan o se han realizado la profesión relacionada con salud mental. *Damos por hecho que usted es enfermera/o y que trabaja o ha trabajado en contacto con salud mental. Si no es así, no debería rellenar este formulario.

Selecciona todos los que correspondan.

Unidad de Hospitalización Breve o Aguda (UHB).

Centro de Atención Integral a Drogodependientes (CAID).

Unidad Residencial.

Unidad de rehabilitación.

Hospital de Día adultos.

Hospital de Día Infanto-Juvenil.

Centro de Salud Mental (CSM).

Otro:

\section{7. ¿Has obtenido la especialidad en salud mental? *}

Marca solo un óvalo

Sí, soy enfermera/o especialista en salud mental. Pasa a la pregunta 8.

No, soy enfermera en contacto con salud mental. Pasa a la pregunta 9.

\section{Enfermera especialista en salud mental.}

En caso de tratarse de un enfermero especialista en salud mental, usted deberá contestar esta pregunta.

\section{8. ¿Cómo obtuvo el título de la especialidad? *}

Marca solo un óvalo

Vía enfermero interno residente (EIR). Pasa a la pregunta 9.

Vía extraordinaria. Pasa a la pregunta 9.

Pasa a la pregunta 9.

\section{Cuestionario.}

Muchas gracias por la información. Ahora comienzan las preguntas propias del cuestionario.

\section{Por favor, lea las siguientes frases acerca de José:}

José es un soltero de 30 años con esquizofrenia. A veces oye voces y se altera. Vive solo en un apartamento y trabaja como empleado en una gran firma de abogados. Ha sido hospitalizado 6 veces a causa de su enfermedad.

AHORA CONTESTE CADA UNA DE LAS SIGUIENTES PREGUNTAS SOBRE JOSÉ. MARCA EL NÚMERO DE LA MEJOR RESPUESTA PARA CADA PREGUNTA. 
9. 1. Me sentiría violentado a causa de José. * Marca solo un óvalo

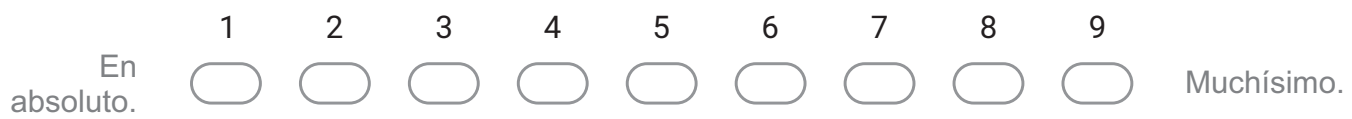

10. 2. Me sentiría inseguro cerca de José. * Marca solo un óvalo

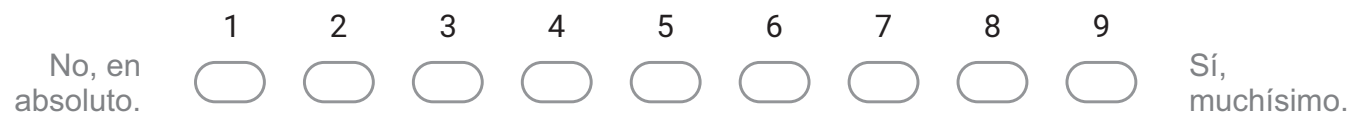

11. 3. José me aterraría. *

Marca solo un óvalo

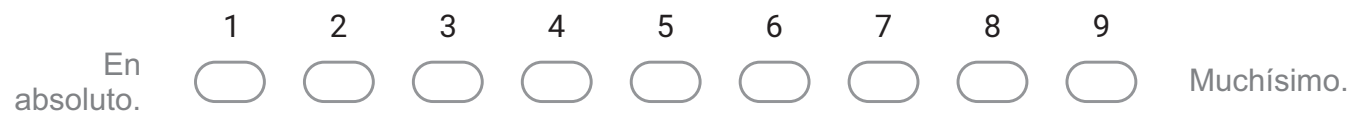

12. 4. ¿En qué medida se sentiría enfadado con José? * Marca solo un óvalo

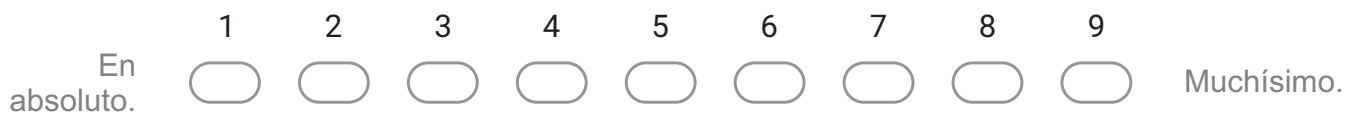

13. 5. Si estuviera al cargo del tratamiento de José, le exigiría que se tomara su medicación. * Marca solo un óvalo

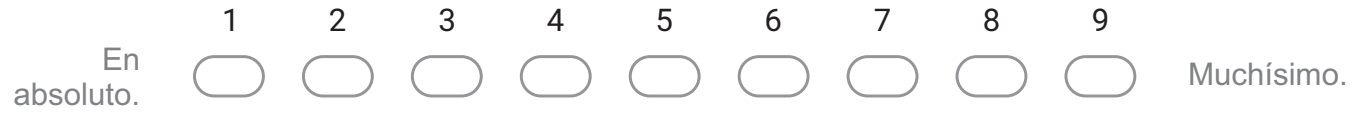

14. 6. Creo que José supone un riesgo para sus vecinos si no se le hospitaliza. * Marca solo un óvalo

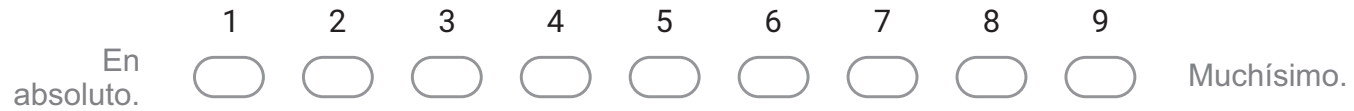


15. 7. Si fuera un empresario, entrevistaría a José para un trabajo * Marca solo un óvalo

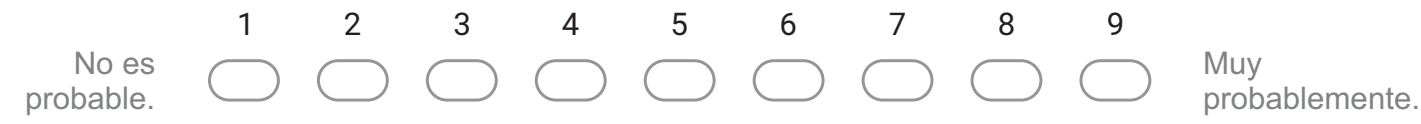

16. 8. Estaría dispuesto a hablar con José acerca de su problema. * Marca solo un óvalo

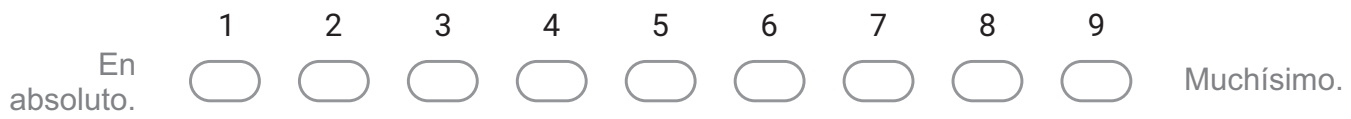

17. 9. Sentiría pena por José. *

Marca solo un óvalo

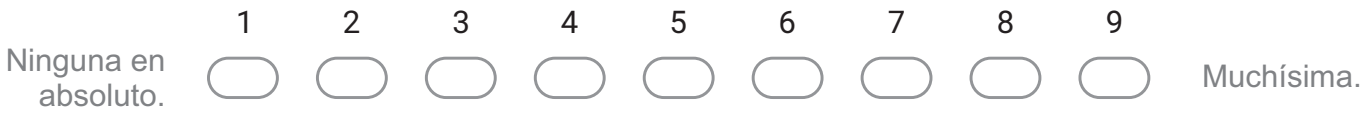

18. 10. Pensaría que es culpa de José que se encuentre en su situación actual * Marca solo un óvalo

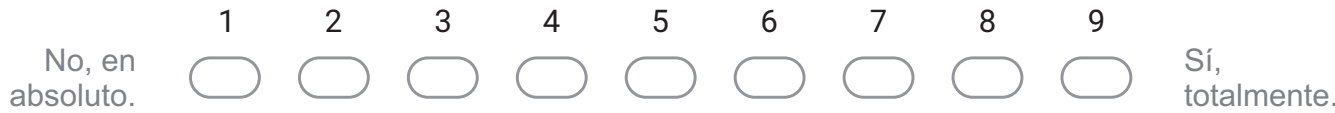

19. 11. ¿Hasta qué punto crees que es controlable la causa de la situación actual de José? * Marca solo un óvalo

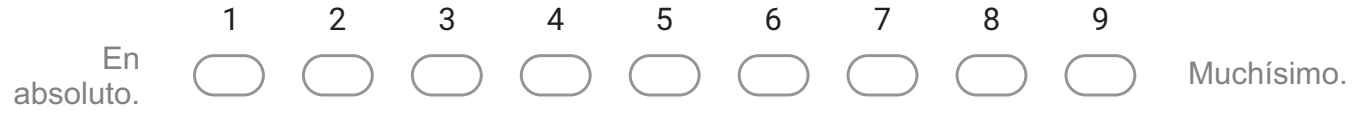

20. 12. ¿En qué medida se sentiría irritado por José? *

Marca solo un óvalo

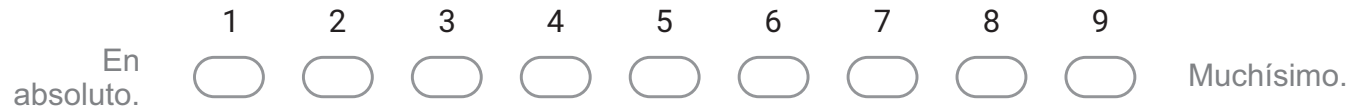


21. 13. En tu opinión, ¿hasta qué punto es José peligroso? * Marca solo un óvalo

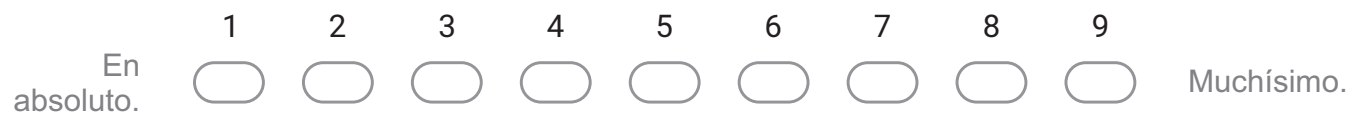

22. 14. ¿Hasta qué punto está de acuerdo en que José debe ser obligado a ponerse en tratamiento médico incluso si él no quiere? *

Marca solo un óvalo

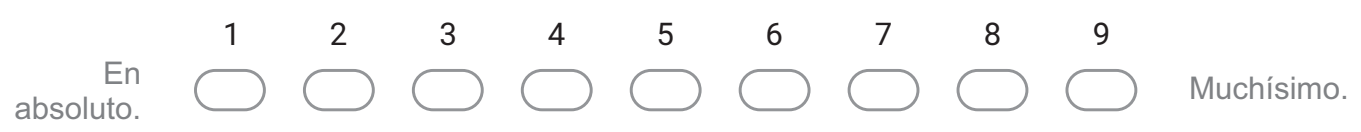

23. 15. Sería mejor para la comunidad de José que lo recluyeran en un hospital psiquiátrico. * Marca solo un óvalo

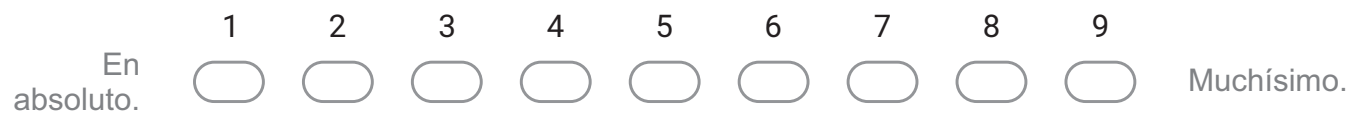

24. 16. Compartiría el coche para ir al trabajo con José cada día. * Marca solo un óvalo

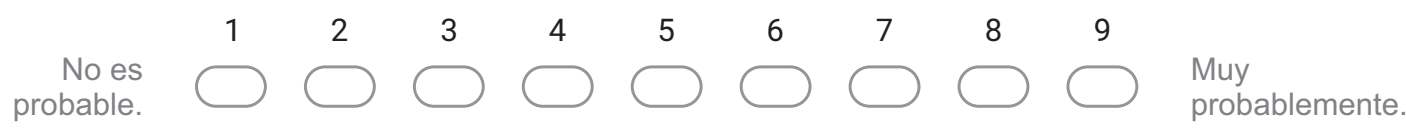

25. 17. ¿Hasta qué punto cree que un hospital psiquiátrico, donde José pudiera mantenerse alejado de sus vecinos, es el mejor lugar para él? *

Marca solo un óvalo

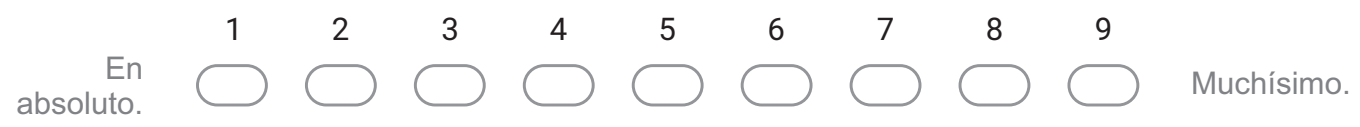

26. 18. Me sentiría amenazado por José. * Marca solo un óvalo

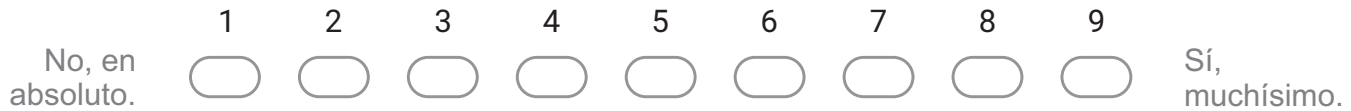


27. 19. ¿Hasta qué punto José le asustaría? * Marca solo un óvalo

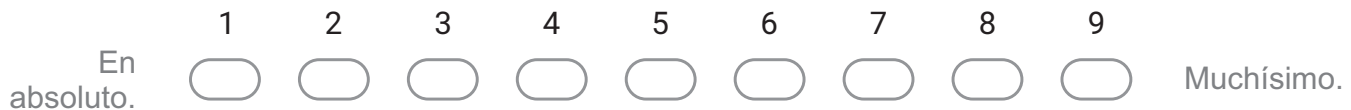

28. 20. ¿Cuál es la probabilidad de que ayudara a José? * Marca solo un óvalo

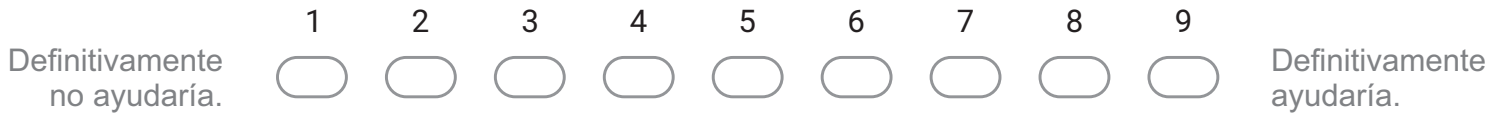

29. 21. ¿Con qué certeza cree que ayudaría a José? * Marca solo un óvalo

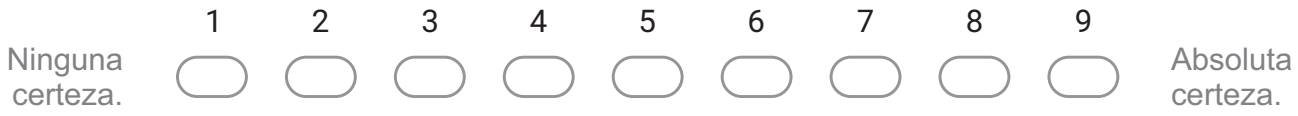

30. 22. ¿Hasta qué punto comprende a José? * Marca solo un óvalo

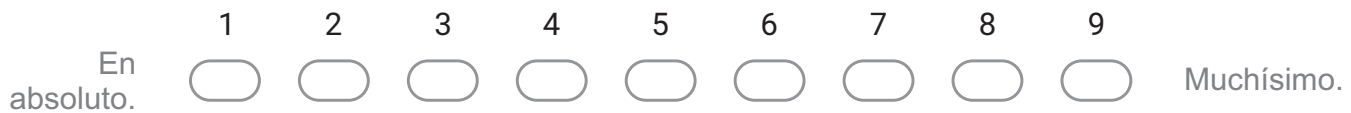

31. 23. En su opinión, ¿hasta qué punto es José responsable de su situación actual? * Marca solo un óvalo

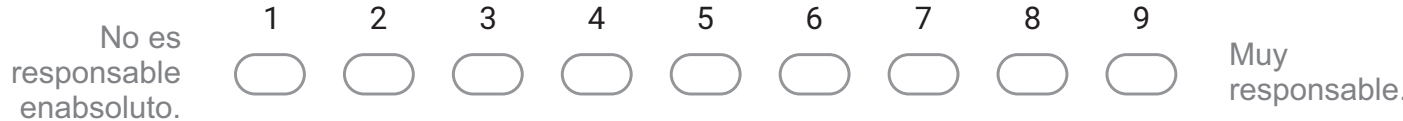

32. 24. ¿Hasta qué punto le aterrorizaría José?. * Marca solo un óvalo

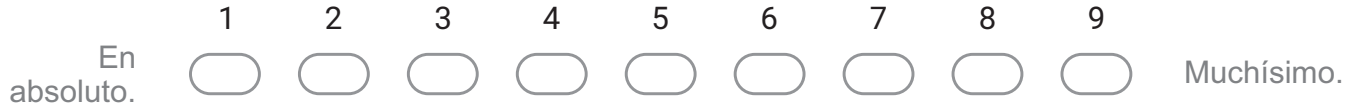


33. 25. Si estuviera al cargo del tratamiento de José, le obligaría a vivir en un piso supervisado. * Marca solo un óvalo
En
$\overbrace{}^{2}$
$\overbrace{}^{3}$
$\overbrace{}^{4}$
${ }^{5}$
$\overbrace{}^{6}$
$\overbrace{}^{7}$
$\bigcirc^{8}$
Muchísimo.

34. 26. Si fuera un propietario, probablemente alquilaría un apartamento a José. * Marca solo un óvalo

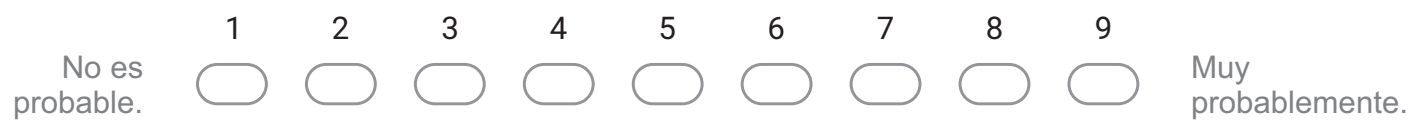

35. 27. ¿Cuánta preocupación sentiría por José? *

Marca solo un óvalo

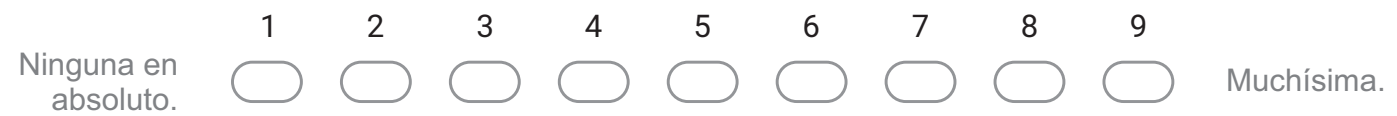

